\title{
Dynamic versus rigid stabilization for the treatment of disc degeneration in the lumbar spine
}

Authors Samo K Fokter, Andrej Strahovnik

Institution Department for Orthopaedic Surgery and Sports Trauma, Celje Teaching Hospital, Celje, Slovenia

\begin{tabular}{l|l|}
\hline $\begin{array}{l}\text { Final class of evidence } \\
\text { (CoE)-treatment }\end{array}$ & Yes \\
\hline Study design: & \\
\hline Prospective cohort & \\
\hline Retrospective cohort & \\
\hline Case control & \\
\hline Case series & \\
\hline Methods & \\
\hline Patients at similar point in \\
course of treatment \\
\hline Follow-up $\geq 85 \%$ \\
\hline Similarity of treatment \\
protocols for patient groups \\
\hline Patients followed-up long \\
enough for outcomes to occur
\end{tabular}

\section{ABSTRACT}

Study design: Retrospective cohort study.

Clinical question: This study aimed to describe the outcome of stabilization surgery with dynamic instrumentation for degenerative disc disease. The results were compared with age- and gender-matched peers treated with traditional fusion with rigid instrumentation. If necessary, additional nerve elements decompression was undertaken in both groups.

Methods: This study analyzed the success rates of 25 patients aged 47.4 years (mean 95\% confidence interval: 43.1-51.7) treated with stabilization of the involved vertebral dynamic unit(s) with either dynamic or rigid instrumentation with or without additional decompression. Clinical outcome was assessed with Oswestry disability index (ODI) and visual analogue scale (VAS) for back pain, leg pain, and activity level. Satisfaction outcome was measured with Stauffer and Coventry overall satisfaction criteria and VAS for satisfaction. Health-related quality of life was estimated with Short Form-36 (SF-36) questionnaires. Fusion rate and adjacent level(s) was checked with x-ray. Complications recorded in patients' files were evaluated and revision surgeries were stated as treatment failures.

Results: At the 4-year follow-up (range, 2-5 years) significant improvement was noted on some subjective parameters in both groups. No statistical differences were seen between the groups at final follow-up. Five patients (42\%) in the rigid group and two patients $(20 \%)$ in the dynamic group were rated good or excellent according to the overall Stauffer and Coventry satisfaction criteria. Radiologically, seven patients $(58 \%)$ in the rigid group were undoubtedly fused and all the involved discs in the dynamic group continued to degenerate. Adjacent segments showed loss of disc height in both groups but only loss of upper adjacent discs in the rigid group was statistically significant. Two patients in the dynamic group and one patient in the rigid group required reoperation because of the pedicle screw misplacement.

None of the authors or the department with which they are affiliated has received anything of value from or owns stock in a commercial company or institution related directly or indirectly to the subject of this article.

Device(s) status: Approved. 
Conclusion: The results of this study indicate no significant difference between dynamic and rigid stabilization of the lumbar spine for patients with degenerative disc disease (DDD). However, the study is underpowered and further studies on larger and homogeneous group of patients should be undertaken.

\section{STUDY RATIONALE AND CONTEXT}

Significant disagreement exists among spine surgeons regarding the optimal technique of stabilization for treatment of degenerative disorders of the lumbar spine. Dynamic stabilization is believed to reduce progressive degenerative disc disease and prevent adjacent segment degeneration in selected patients with low back pain [1-3].

\section{CLINICAL QUESTION}

The objective was to compare dynamic stabilization versus traditional rigid fusion of the lumbar spine with respect to (1) medium-term clinical outcome, (2) patient satisfaction, and (3) radiological outcome.

\section{METHODS}

Study design: Retrospective cohort study.

Inclusion criteria: Consecutive patients with DDD requiring stabilization of the degenerated segment(s) with or without additional decompressive surgery using either dynamic or rigid instrumentation, operated between February 2006 and February 2009.

Exclusion criteria (Fig 1): Patients with deformities, eg, spondylolisthesis or scoliosis, and without preoperative documentation available at the final follow-up.

\section{Patient population and intervention compared (Fig 1)}

- Of 25 patients who were assessed for eligibility, two from the dynamic and one from the rigid groups were operated again because of transpedicular screw misplacement, and were hence regarded as failures and excluded from the follow-up analysis.

- Treatment was assigned based on surgeon preference and was not consciously related to factors that may have influenced the outcome.

- Dynamic stabilization was accomplished using a dorsal transpedicular device (Dynesys, Centerpulse,
Winterthur, Switzerland). Rigid stabilization was obtained by transpedicular screw fixation (XIA, Stryker Spine, Allendale, New Jersey, USA) and two interbody cages (Stryker Spine, Cestas, France) per segment.

\section{Outcomes}

- Clinical outcomes: Clinical outcome was assessed with Oswestry disability index (ODI), visual analogue scale (VAS) for back pain, leg pain, and activity level, filled in by patients preoperatively and at follow-up.

- Satisfaction outcomes: Stauffer and Coventry overall satisfaction criteria and VAS for satisfaction of the treatment were taken at follow-up.

- Health-related quality of life: Health-related quality of life was estimated with a Short Form-36 (SF-36) questionnaire at follow-up.

- Radiological outcomes: Signs of fusion and disc height of the involved and adjacent segment(s) were determined on the standing lateral $x$-rays. Standing lateral $\mathrm{x}$-rays of the lumbar spine were evaluated preoperatively and at follow-up. Plain x-rays were taken between 3 and 6 hours after the patient woke up in the morning. The central x-ray beam was focused on L4. X-rays with evident projectional distortions due to lateral tilt or longitudinal rotation were excluded, and new x-rays were taken from the same patient. The disc height was calculated as the mean of the anterior, middle, and posterior disc heights according to the criteria of Quint et al [4]. Solid fusion was determined to be present only if a sentinel was evident.

- Complications and treatment failures: All complications recorded in patients' files were thoroughly evaluated. Revision surgeries were stated as treatment failures.

\section{Analysis}

- Clinical outcomes are presented in median values, with interquartile ranges within parentheses.

- Intra-group changes from preoperative to followup values were compared using a Wilcoxon signed rank test. Changes between groups were compared with a Mann-Whitney test. Differences in categorical baseline variables (eg, gender) were tested using a Fisher exact test and a chi-square test (if contingency table consisted of more than $2 \times 2$ cells).

- Data were analyzed using the SPSS 17.0 statistical software (SPSS Inc, Chicago, Illinois, USA). $P$ values $<.05$ were considered statistically significant. 


\section{RESULTS}

- The two groups did not differ significantly at baseline with respect to variables that were measured (Table 1). The follow-up time for the dynamic group was 38 months (median range, 21-60 months), and for the rigid group 53.5 months (median range, 25-60 months).

- Clinical outcomes: There were no significant differences between the groups with regard to the change in preoperative and follow-up values of ODI, VAS back pain, VAS leg pain, and VAS activity level (Table 2). Significant improvements (preoperative versus followup values) were seen in VAS back pain, VAS leg pain, and VAS activity level in both groups; and ODI in the dynamic group (Table 2).

- Satisfaction outcomes: Patients in both groups were equally satisfied with the performed treatment (VAS $=7$ in both groups, $P=.61$ ). According to Stauffer and Coventrys overall satisfaction criteria, grades excellent or good were achieved for two patients $(20 \%)$ in the dynamic group and for five patients $(42 \%)$ in the rigid group $(P=.25)$.

Table 1 Demographic and surgical baseline characteristics.

\begin{tabular}{llll}
\hline & $\begin{array}{l}\text { Dynamic } \\
\text { group } \\
(\mathbf{n = 1 2 )}\end{array}$ & $\begin{array}{l}\text { Rigid } \\
\text { group } \\
(\mathbf{n = 1 3 )}\end{array}$ & P value \\
\hline Age, y (interquartile ranges) & $47.5(15)$ & $52(20)$ & $.72^{*}$ \\
\hline Female, No. (\%) & $6(50)$ & $6(46)$ & $1.00 \dagger$ \\
\hline No. of treated segments (one:two) & $9: 3$ & $11: 2$ & $.65 \dagger$ \\
\hline Body mass index, kg/m² & $27.7(8)$ & $30(8)$ & $.28^{*}$ \\
\hline Treated levels, \# & & & $.52 \ddagger$ \\
L3-L4 & 2 & 0 & \\
L4-L5 & 6 & 9 & \\
L5-S1 & 1 & 2 & \\
L3-L5 & 1 & 1 & \\
L4-S1 & 2 & 1 & \\
\hline Reason for surgery, \# & & & \\
Degenerative disc disease (DDD) & 3 & 3 & \\
DDD + disc herniation with sciatica & 4 & 3 & \\
DDD + spinal stenosis with & 4 & 5 & \\
claudication & 1 & 2 & \\
DDD + previous disc surgery & 1 & 2 & \\
\hline
\end{tabular}

* Mann-Whitney test.

$\dagger$ Fisher exact test.

‡ Chi-square test.
- Health-related quality of life: No significant differences were seen in any of the SF-36 parameters (Table 4) (Fig 2).

- Radiological outcomes: The involved discs in the dynamic group continued to degenerate (Table 3) (Fig 3a-b). Adjacent segments showed loss of disc height in both groups but only loss of upper adjacent discs in the rigid group was statistically significant (Table 3). Solid fusion was undoubtedly determined in seven patients $(58 \%)$ from the rigid group. No obvious signs of non-fusion, eg, screw breakage or loosening, were noted on x-rays.

- Complications and treatment failures: No surgical complications, eg, dural tear, nerve root lesion, wound infection or hematoma was recorded in any patient. However, two patients in the dynamic group and one patient in the rigid group required reoperation because of the pedicle screw misplacement resulting in nerve root irritation and pain. After revision surgeries with rigid instrumentation, all fusions were solid with positive sentinel sign on $\mathrm{x}$-ray at final follow-up.

Table 2 Intra-group and inter-group changes of clinical outcomes.

\begin{tabular}{|c|c|c|c|c|}
\hline & \multicolumn{2}{|l|}{ ODI } & \multicolumn{2}{|c|}{$\begin{array}{l}\text { Back pain } \\
\text { (VAS) }\end{array}$} \\
\hline & $\begin{array}{l}\text { Dynamic } \\
(\mathrm{N}=10)\end{array}$ & $\begin{array}{l}\text { Rigid } \\
(\mathrm{N}=12)\end{array}$ & $\begin{array}{l}\text { Dynamic } \\
(\mathrm{N}=10)\end{array}$ & $\begin{array}{l}\text { Rigid } \\
(\mathrm{N}=12)\end{array}$ \\
\hline Baseline, points (IQR) & $49.5(37)$ & $66(14)$ & $7.5(2)$ & $9(2)$ \\
\hline Follow-up, points (IQR) & $47(42)$ & $40(18)$ & $5.5(2)$ & $6(3)$ \\
\hline Median change, points (IQR) & $-9(36)$ & $-26(15)$ & $-1.5(4)$ & $-2(4)$ \\
\hline $\begin{array}{l}\text { Within group } P \text { value for } \\
\text { change score }^{\dagger}\end{array}$ & .16 & .02 & .02 & .02 \\
\hline \multirow{3}{*}{$\begin{array}{l}\text { Between group } P \text { value for } \\
\text { change score }{ }^{\ddagger}\end{array}$} & .15 & & 1.0 & \\
\hline & \multicolumn{2}{|c|}{$\begin{array}{l}\text { Leg pain } \\
\text { (VAS) }\end{array}$} & \multicolumn{2}{|c|}{$\begin{array}{l}\text { Activity level } \\
\text { (VAS) }\end{array}$} \\
\hline & $\begin{array}{l}\text { Dynamic } \\
(\mathrm{N}=10)\end{array}$ & $\begin{array}{l}\text { Rigid } \\
(\mathrm{N}=12)\end{array}$ & $\begin{array}{l}\text { Dynamic } \\
(\mathrm{N}=10)\end{array}$ & $\begin{array}{l}\text { Rigid } \\
(\mathrm{N}=12)\end{array}$ \\
\hline Baseline, points (IQR) & $9(2)$ & $8(2)$ & $1.5(3)$ & $1.5(1)$ \\
\hline Follow-up, points (IQR) & $5(2)$ & $5(4)$ & $4.5(2)$ & $5(4)$ \\
\hline $\begin{array}{l}\text { Median change, } \\
\text { points (IQR) }\end{array}$ & $-4(2)$ & $-3(4)$ & $2.5(3)$ & $4(3)$ \\
\hline $\begin{array}{l}\text { Within group } P \text { value for } \\
\text { change scoref }\end{array}$ & $<.01$ & .03 & .02 & $<.01$ \\
\hline $\begin{array}{l}\text { Between group } P \text { value for } \\
\text { change score } \neq\end{array}$ & .18 & & .24 & \\
\hline
\end{tabular}

* ODI indicates Oswestry disability index; VAS, visual analogue scale; and $\mathrm{IQR}$, interquartile ranges.

† Wilcoxon signed ranks test (exact).

$\ddagger$ Mann-Whitney test (exact). 
Table 3 X-ray measurements comparing treatment groups.

\begin{tabular}{|c|c|c|c|c|c|}
\hline & & Preoperatively & Follow-up & Median change & $P$ value \\
\hline \multirow[t]{3}{*}{$\begin{array}{l}\text { Involved disc height, median, mm } \\
\text { (interquartile ranges [IQR]) }\end{array}$} & $\begin{array}{l}\text { Dynamic group } \\
\mathrm{N}=13^{\dagger}\end{array}$ & $9(4)$ & $6(3)$ & $2(2)$ & $<.01^{*}$ \\
\hline & $\begin{array}{l}\text { Rigid group } \\
\mathrm{N}=13^{\dagger}\end{array}$ & $10.3(5)$ & $12.5(2)$ & $-2(4)$ & $<.01 *$ \\
\hline & Between group $P$ value $\ddagger$ & & & $<.01$ & \\
\hline \multirow[t]{3}{*}{$\begin{array}{l}\text { Upper adjacent disc height, median, mm } \\
\text { (IQR) }\end{array}$} & $\begin{array}{l}\text { Dynamic group } \\
N=10\end{array}$ & $10.5(3)$ & $10(2)$ & $0(1)$ & $.13^{*}$ \\
\hline & $\begin{array}{l}\text { Rigid group } \\
\mathrm{N}=12\end{array}$ & $13(3)$ & $12.5(3)$ & $1.5(2)$ & $.02 *$ \\
\hline & Between group $P$ value ${ }^{\ddagger}$ & & & .10 & \\
\hline \multirow[t]{3}{*}{$\begin{array}{l}\text { Lower adjacent disc height, median,mm } \\
\text { (IQR) }\end{array}$} & $\begin{array}{l}\text { Dynamic group } \\
\mathrm{N}=8^{\dagger}\end{array}$ & $6.5(4)$ & $5.5(2)$ & $0.5(3)$ & $.13^{*}$ \\
\hline & $\begin{array}{l}\text { Rigid group } \\
\mathrm{N}=10^{\dagger}\end{array}$ & $14(7)$ & $12(6)$ & $1(3)$ & $.06^{*}$ \\
\hline & Between group $P$ value ${ }^{\ddagger}$ & & & .67 & \\
\hline Successful fusion & Rigid group & Not applicable & 7 & & \\
\hline
\end{tabular}

* Wilcoxon signed ranks test (exact).

$\dagger$ The number of involved discs differs from number of patients since three patients (of 10 analyzed) in the dynamic group and one patient (of 12 analyzed) in the rigid group had two operated segments. Additionally, the number of lower adjacent discs also differs according to the operated level (no lower disc if L5-S1 is the involved segment).

₹ Mann-Whitney test (exact).

Fig 1 Patient sampling and selection.

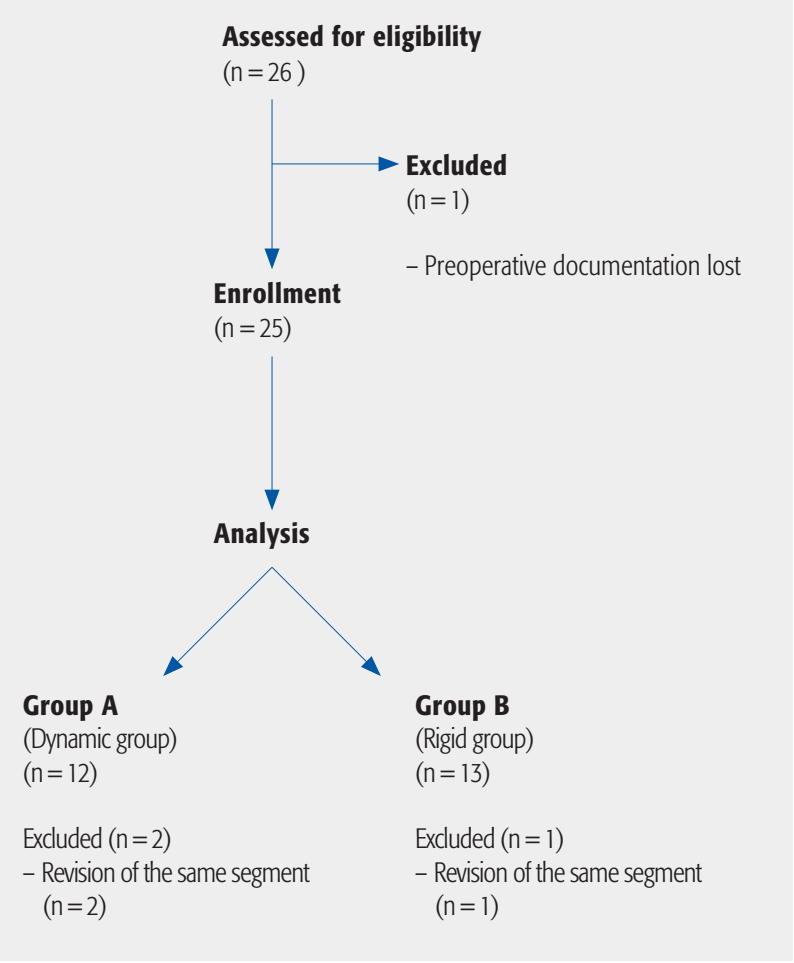


Table 4 Mean and $P$ values of the follow-up SF-36 questionnaire.

\begin{tabular}{lllllllllll}
\hline & PF & RP & BP & GH & VT & SF & RE & MH & PCS & MCS \\
\hline Dynamic group & 34.1 & 31.1 & 33.3 & 35.8 & 45.8 & 29.6 & 27.5 & 37.8 & 32.0 & 35.9 \\
\hline Rigid group & 26.7 & 28.0 & 34.8 & 32.2 & 50.2 & 36.7 & 27.5 & 38.4 & 30.1 & 40.9 \\
\hline Pvalue & .22 & .67 & .49 & .81 & .13 & .06 & .94 & .40 & .78 & .24 \\
\hline
\end{tabular}

$\mathrm{PF}=$ physical functioning; $\mathrm{RP}=$ role $=$ physical; $\mathrm{BP}=$ bodily pain; $\mathrm{GH}=$ general health; $\mathrm{VT}=$ vitality; $\mathrm{SF}=$ social functioning; $\mathrm{RE}=$ role $=$ emotional $\mathrm{MH}=$ mental health; $\mathrm{PCS}=$ physical component summary; $\mathrm{MCS}=$ mental component summary

Fig 2 SF-36 parameters at follow-up.

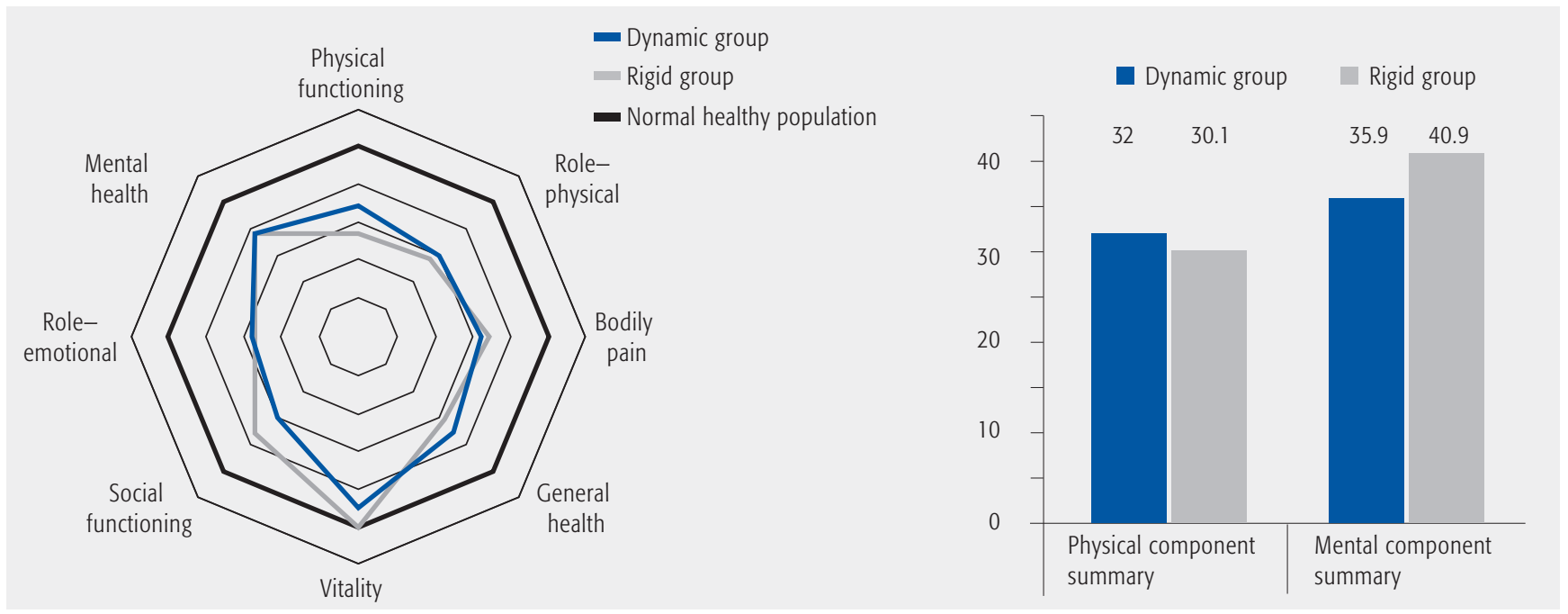

Fig 3a Continued degeneration of the involved L4-L5 disc before treatment.

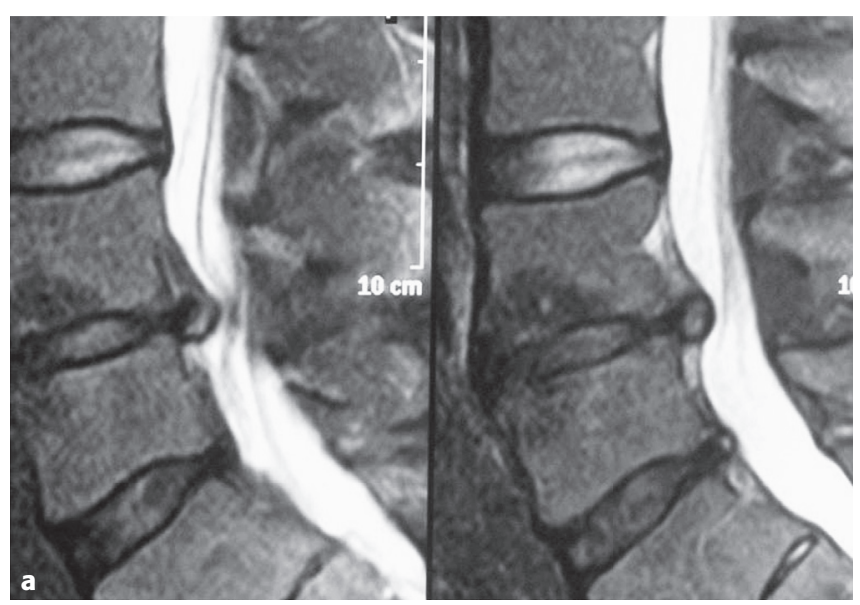

Fig 3b Continued degeneration of the involved L4-L5 disc 3 years after discectomy and dynamic stabilization.

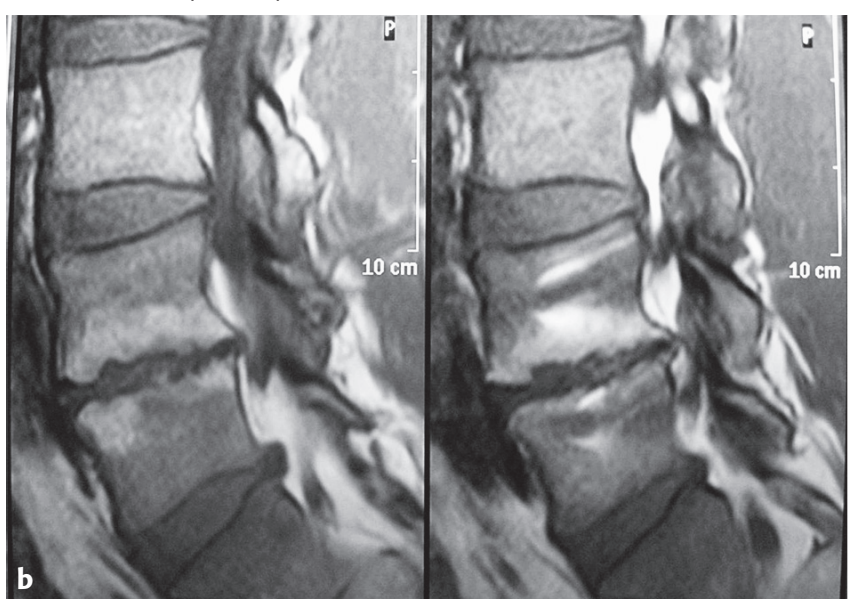




\section{DISCUSSION}

- Regarding clinical outcomes, significant improvements in back pain, leg pain and activity level were obtained in patients with either dynamic or rigid stabilization of the lumbar spine.

- Another retrospective multicenter study [5] has shown significantly improved mean back and leg pain as well as ODI scores from the baseline to 12-month followup evaluation in patients requiring decompression and instrumented fusion for one or two continuous spinal levels treated with the Dynesys construct. On the contrary, our study was not able to demonstrate any significant improvement in the ODI score at the 4-year follow-up.

- There does not appear to be a clear clinical advantage of dynamic over rigid stabilization with regard to patient satisfaction as measured by VAS and health-related quality of life as measured with SF-36. However, with regard to overall satisfaction criteria, patients treated with rigid stabilization have shown better results.

- Regarding radiological outcomes, our results have shown continued disc degeneration of the dynamic group at the treated level where disc height has decreased by $2 \mathrm{~mm}$ on the average. This was statistically significant. Adjacent segment degeneration was also noted in both groups but was statistically significant for the upper adjacent segment of the rigid group only, where disc height has decreased by $1.2 \mathrm{~mm}$ on the average. This fact may be attributed to the small number of patients.

- According to a recent x-ray analysis of lumbar spine range of motion after monosegmental fusion and posterior dynamic stabilization, Cakir et al [6] were unable to show any effect of Dynesys or of rigid fusion with regard to adjacent segment mobility. Our results have confirmed adjacent segment degeneration in both groups, which may be attributed to the normal or accelerated aging process of the lumbar spine.

- Regarding complications, reoperation rate was somewhat higher in the dynamic group and was mostly because of the pedicle screw misplacement. This may be linked to the slightly different entry point of the dynamic instrumentation unfamiliar to the traditional spine surgeon, larger self-cutting screws, and/or simply the learning curve.

- In one retrospective study of patients consecutively treated by Dynesys for DDD and evaluated with a postal, patient-oriented follow-up questionnaire [7], the authors were unable to provide support for the notion that dynamic fixation of the lumbar spine results in better outcomes than those typical of fusion. Besides, the reoperation rate after Dynesys was relatively high $(19 \%)$ at 2-year follow-up. This is consistent with results of our study, in which two of 12 patients required reoperation and fusion and one was scheduled for a further surgical intervention, giving the reoperation rate of $25 \%$.

- Strengths: Ours is a single-center controlled trial where potential differences between comparison groups other than those related to fixation were kept to a minimum.

- Limitations: The number of patients in each group was small and the study has been underpowered to detect statistical differences between groups. The difference in ODI change (follow-up - baseline values) between groups was clinically relevant (> 10 [8]). However, if we wanted for the difference in ODI change to be statistically significant, we would need 80 patients in each group $(\alpha=0.05,1-\beta=0.8)$. Moreover, the patient population was not homogeneous as surgeries were performed for different indications and on different levels which may have biomechanical consequences. Besides, the median follow-up time was 13.5 months shorter in the dynamic group which may also influence the results. A computed tomographic analysis is better for radiological assessment of fusion but it would further burden the patients with radiation exposure.

\section{SUMMARY AND CONCLUSION}

- This is one of the few controlled studies to compare the clinical and x-ray outcomes of two different types of fixation (one dynamic and one rigid) in patients who would benefit from surgical stabilization for the DDD with a medium-term follow-up.

- We did not observe a significant difference between the two techniques regarding patient-oriented and radiological outcomes, but acknowledge that the study is underpowered.

- Revision rate was higher in the dynamic group but this may be due to the small number of patients.

- Additional randomized studies with larger sample sizes, homogeneous patient population, and homogeneous treatment options are needed to further evaluate the benefits of dynamic versus rigid stabilization of the lumbar spine. 


\section{REFERENCES}

1. Stoll TM, Dubois G, Schwarzenbach 0 (2002) The dynamic neutralization system for the spine: a multi-center study of a novel non-fusion system. Eur Spine J; 11(suppl 2):170-178.

2. Putzier M, Schneider SV, Funk JF, et al (2005) The surgical treatment of the lumbar disc prolapse: nucleotomy with additional transpedicular dynamic stabilization versus nucleotomy alone. Spine; 30(5):E109-E114.

3. Schnake KJ, Schaeren S, Jeanneret B (2006) Dynamic stabilization in addition to decompression for lumbar spinal stenosis with degenerative spondylolisthesis. Spine; 31(4):442-449.

4. Quint DJ, Tuite GF, Stern JD, et al (1997) Computer-assisted measurement of lumbar spine radiographs. Acad Radiol; 4(11):742-752.

5. Welch WC, Cheng BC, Awad TE, et al (2007) Clinical outcomes of the Dynesys semirigid neutralization system: 1-year preliminary results. Neurosurg Focus; 22(1):E8.

6. Cakir B, Carrazo C, Schmidt R, et al (2009) Adjacent segment mobility after rigid and semirigid instrumentation in the lumbar spine. Spine; 34(12):1287-1291.

7. Grob D, Benini A, Junge A, et al (2005) Clinical experience with the Dynesys semirigid fixation system for the lumbar spine: surgical and patient-oriented outcome in 50 cases after an average of 2 years. Spine; 30(3):324-331.

8. Ostelo RW, de Vet HC (2005) Clinically important outcomes in low back pain. Best Pract Res Clin Rheumatol; 19(4):593-607.

\section{EDITORIAL PERSPECTIVE}

The reviewers congratulated Fokter and Strahovnik on tackling an interesting and controversial subject with a well-designed study. They make a good effort to compare the two groups clinically and radiographically. Dealing with degenerative lumbar spine conditions notoriously opens the door for a myriad of variables and confounding factors, which make real-life research in this area so very challenging.
(1) The reviewers joined the authors in noting that this retrospective study comparing 12 patients in one arm with 13 patients in another arm was underpowered due to lack of sample size. However, with the lack of a power, it is not accurate to conclude that there is no difference or even that "there does not appear to be a clear clinical advantage" of one technique over the other. Therefore, to conclude a null hypothesis is inaccurate. Rather, the findings simply support that the authors did not observe a significant difference between the two techniques, while acknowledging that the study is underpowered.

(2) The inclusion criteria are patients with "degenerative disc disease [DDD] requiring decompressive surgery with additional stabilization of the degenerated segment(s)." Fokter and Strahovnik list the number of patients with DDD only and DDD with herniated disc, stenosis, and prior disc surgery. This inclusion, however, is broad and includes many variables, and does not exclude deformity such as scoliosis or spondylolisthesis. The study group includes patients who underwent "decompression" for presumptive neurocompressive pathology. It is impossible to point to how much the improvement of symptoms in both groups is attributable to decompression of the neural elements versus function of the implants. In the future, they may want to consider the two groups in patients with DDD who did not require decompression. Thus, the effect of these implants without a concurrent decompression can better be assessed.

(3) The fusion rate as $x$-ray outcomes remains controversialfusions may have occurred in the dynamic group and may not have been identified. Conversely, the 58\% fusion rate in the rigid group after more than 2 years seems very low and raises questions about the surgical techniques.

(4) An interesting biomechanical observation involved the levels of instrumentation. The consequences of a L5-S1 and a L4-L5 stabilization are not comparable. Because after a lumbosacral stabilization, there is one adjacent segment left compared with two adjacent segments for all other segments.

(5) Finally, one of the reviewers challenged the indication for dynamic stabilization shown in Figure 3.

In light of a degenerated and extruded disc, with significant decrease of the disc height in L4/5 and a degenerated disc in L5/S1, the reviewer believed that the L4/5 was too unstable for dynamic stabilization and would have recommended a rigid fusion.

These points are helpful in advancing our understanding toward creating better future studies. Fokter and Strahovnik deserve praise for an excellent effort and simulating valuable further discussions on this surgical technique, and how we can improve our research efforts. 